\title{
Clinical Outcome of Hyperuricemia in IgA Nephropathy: A Retrospective Cohort Study and Randomized Controlled Trial
}

\author{
Yongjun Shi ${ }^{\mathrm{a}}$ Wei Chen ${ }^{\mathrm{a}}$ Diana Jalal ${ }^{\mathrm{d}}$ Zhibin $\mathrm{Li}^{\mathrm{a}, \mathrm{b}}$ Wenfang Chen ${ }^{\mathrm{c}}$ \\ Haiping Mao a Qiongqiong Yang ${ }^{a}$ Richard J. Johnson ${ }^{d}$ Xueqing $\mathrm{Yu}^{\mathrm{a}}$ \\ Department of a Nephrology and ${ }^{b}$ Epidemiology Research Unit, Translational Medicine Research Centre, and \\ 'Department of Pathology, The First Affiliated Hospital, Sun Yat-sen University, Guangzhou, China; ${ }^{\mathrm{d}}$ Division of \\ Renal Diseases and Hypertension, University of Colorado Denver, Aurora, Colo., USA
}

\section{Key Words}

Hyperuricemia $\cdot \lg$ A nephropathy $\cdot$ Prognosis $\cdot$ Outcome

\begin{abstract}
Background: Hyperuricemia is an independent risk factor for renal progression in IgA nephropathy (IgAN). However, no study has evaluated the effect of allopurinol on the clinical outcome in hyperuricemic IgAN. Methods: First, a retrospective cohort study of $353 \mathrm{lgAN}$ patients was conducted to explore the relationship between uric acid (UA) and the progression of renal disease over a mean period of 5 years. Then, 40 hyperuricemic IgAN patients were randomized to receive allopurinol (100-300 mg/day) or usual therapy for 6 months. The study outcomes were renal disease progression and/or blood pressure. Results: Hyperuricemia independently predicted renal survival at 1,3 , and 5 years after adjustment for different baseline estimated glomerular filtration rates. In the randomized controlled trial, allopurinol did not significantly alter renal progression or proteinuria. The antihypertensive drug dosage was reduced in 7 of 9 cases with hypertension in the allopurinol group compared to 0 of 9 cases in the control group $(p<0.01)$. UA levels correlated with mean arterial pressure in normotensive patients $(r=$ $0.388, p<0.001$ ). Conclusion: Hyperuricemia predicts the progression of IgAN independently of baseline estimated
\end{abstract}

\section{KARGER}

(C) 2011 S. Karger AG, Basel

Fax +41613061234 E-Mail karger@karger.ch www.karger.com www.karger.com/kbr glomerular filtration rate. Allopurinol may improve the control of blood pressure. Further studies are required to explore the effects of lowering UA on renal protection in IgAN.

Copyright $\odot 2011$ S. Karger AG, Basel

\section{Introduction}

IgA nephropathy (IgAN) is the most common primary glomerulonephritis in the world and approximately $30 \%$ of these patients progress to end-stage renal disease. Risk factors for disease progression include male gender, young age at disease onset, absence of episodes of recurrent macrohematuria, persistent microscopic hematuria, hypertension, the extent of proteinuria, baseline renal insufficiency and pathological markers (including global glomerular sclerosis, interstitial fibrosis, and crescent formation) [1].

An elevated serum uric acid (UA) is also a risk factor for progression of renal disease in Finnish and Japanese patients with IgAN [2-4]. Whether hyperuricemia is a risk factor for renal progression in IgAN in China has not been determined, nor is there any data on whether lowering UA with allopurinol can improve renal outcomes in these patients. We therefore firstly conducted a retrospective analysis of 353 subjects with IgAN with a mean 
follow-up period of 5 years to determine the relationship of UA with progression of renal disease. We also performed a pilot randomized trial of $40 \mathrm{Ig} \mathrm{AN}$ patients with hyperuricemia to explore the effect of lowering UA with allopurinol on the preservation of renal function or use of antihypertensive treatment.

\section{Methods}

\section{Retrospective Cohort Study}

The retrospective cohort study was conducted on patients with biopsy-proven IgAN between January of 1993 and December of 2006 at the First Affiliated Hospital of Sun Yat-sen University in Guangzhou, China. IgAN was defined as chronic glomerulonephritis with IgA immunoglobulin as the sole or main glomerular immunofluorescence finding, required the presence of at least 5 glomeruli, and was confirmed by a blinded pathologist using Lee's grading system [5]. Inclusion criteria included biopsy-proven IgAN with an estimated glomerular filtration rate (eGFR) of $\geq 30$ $\mathrm{ml} / \mathrm{min} / 1.73 \mathrm{~m}^{2}$ at the time of renal biopsy (using the Chinese abbreviated MDRD equation [6]). 643 consecutive patients were considered. However, subjects were excluded if they were younger than 18 years at the time of renal biopsy $(n=37)$, if they had a secondary cause of IgA deposition (systemic lupus erythematosus, Henoch-Schönlein purpura, hepatic diseases or lymphoma, etc.) ( $\mathrm{n}=23)$, if the eGFR was $<30 \mathrm{ml} / \mathrm{min} / 1.73 \mathrm{~m}^{2}$ at the time of renal biopsy $(\mathrm{n}=97)$, or if there was less than 12 months of follow-up $(n=133)$. A total of 353 patients were analyzed following these exclusions. The study was approved by The Human Ethics Committees of The First Affiliated Hospital of Sun Yat-sen University.

Clinical data recorded at biopsy included gender, age, body mass index (BMI), blood pressure (BP), 24-hour urinary protein excretion, serum creatinine ( $\mathrm{Scr}$ ), total cholesterol (TC), triglyceride (TG) and serum UA. Hypertension was defined as systolic blood pressure (SBP) $\geq 140 \mathrm{~mm} \mathrm{Hg}$ and/or diastolic blood pressure (DBP) $\geq 90 \mathrm{~mm} \mathrm{Hg}$ or treatment with antihypertensive drugs. Hyperuricemia was defined as serum UA $>7 \mathrm{mg} / \mathrm{dl}(420 \mu \mathrm{mol} / \mathrm{l})$ in men or $>6 \mathrm{mg} / \mathrm{dl}(>360 \mu \mathrm{mol} / \mathrm{l})$ in women [7]. Hypercholesterolemia was defined as TC levels $>5.3 \mathrm{mmol} / \mathrm{l}$ and hypertriglyceridemia as TG levels $>1.7 \mathrm{mmol} / \mathrm{l}$. BMI was calculated as weight divided by height squared and the obesity defined in these Asian subjects as BMI $\geq 25$ [8]. Proteinuria was calculated from a 24 -hour urine collection. Scr, UA, TC, and TG concentrations were measured enzymatically after an overnight fast at the time of biopsy.

Patients were seen in the clinic at a minimum of every 6 months. At each visit the use of medications and laboratory data were recorded. The primary study endpoint was a decline of eGFR $>50 \%$ compared to the level obtained at renal biopsy or the initiation of renal replacement therapy, or death.

\section{Prospective Randomized Controlled Trial}

A prospective, randomized, parallel, open-label, controlled trial was conducted at The First Affiliated Hospital of Sun Yat-sen University from July of 2007 to June of 2009. 40 patients were enrolled based on the following inclusion criteria: $18-70$ years of age, biopsy-proven IgAN, proteinuria between 0.15 and $2.0 \mathrm{~g} / 24 \mathrm{~h}$ with serum albumin level $>3.5 \mathrm{~g} / \mathrm{dl}, \mathrm{Scr}$ level $<3 \mathrm{mg} / \mathrm{dl}, \mathrm{BP}$
$<180 / 100 \mathrm{~mm} \mathrm{Hg}$, and UA $>6 \mathrm{mg} / \mathrm{dl}$ in women and $>7 \mathrm{mg} / \mathrm{dl}$ in men. Patients were excluded if they received prednisone or immunosuppressive drugs within 2 months prior to randomization, if subjects were receiving angiotensin-converting enzyme inhibitors (ACEI) and/or angiotensin receptor blockers (ARB), if there was a past allergy to allopurinol, the presence of active gout within the past 4 weeks, and women who were pregnant or unwilling to use contraception. The study was approved by the Human Ethics Committees of The First Affiliated Hospital of Sun Yat-sen University and was conducted according to the Declaration of Helsinki principles and registered at ClinicalTrials.gov NCT00793585. All participating patients had signed the written informed consent before enrollment.

\section{Randomization and Treatment Protocol}

Randomization was conducted using a computer-generated random allocation sequence table. Allocation concealment was performed by enclosing assignments in sequentially numbered, opaque-closed envelopes. Patients were randomly assigned to either the treatment or the control group. All subjects received health education and were encouraged to adhere to a low-purine diet. Patients in the treatment group received allopurinol 100-300 $\mathrm{mg}$ /day according to the levels of Scr and UA. For those with Scr $<1.5 \mathrm{mg} / \mathrm{dl}(133 \mu \mathrm{mol} / \mathrm{l})$ at the baseline, allopurinol was given 100 $\mathrm{mg}$ three times daily, and when serum UA deceased to the normal range (serum UA level $\leq 6 \mathrm{mg} / \mathrm{dl}$ in females and $\leq 7 \mathrm{mg} / \mathrm{dl}$ in males), allopurinol was changed to $100 \mathrm{mg}$ twice daily. For patients with Scr $\geq 1.5 \mathrm{mg} / \mathrm{dl}$ at baseline, allopurinol was initiated at $100 \mathrm{mg}$ twice daily and was decreased to $100 \mathrm{mg}$ daily when UA decreased into the normal range.

Arterial BP was recorded with a mercury sphygmomanometer and measured according to the Joint National Committee VII criteria (JNC VII) [9]. The mean of three measurements was recorded. Mean arterial pressure (MAP) was calculated from SBP and DBP. Patients diagnosed with hypertension received antihypertensive drugs with titration during the follow-up. The calcium antagonist nifedipine controlled-release tablet was the firstchoice drug and its dose doubled in uncontrolled patients, followed by the addition of either a $\beta$-blocker and (or) $\alpha$-blocker if the patients were still in a hypertensive condition. The therapeutic target of BP was $\leq 130 / 80 \mathrm{~mm} \mathrm{Hg}$. The washout time for the ACEI and ARBs, pulse intravenous corticosteroids, and intravenous immunoglobulin were at least 8 weeks prior to randomization, and all drugs had been mentioned above were prohibited throughout the study. Hyperlipidemia was treated with statins and/or fibric acid derivatives as required.

Follow-Up Assessment

Patients were evaluated every 2 weeks in the first month, and then followed up monthly for a total of 6 months. At each followup the patients underwent clinical evaluation and assessment for adverse effects. BP and laboratory tests including a complete blood count, urinalysis, $U_{\text {prot/creat }}$ ratio, serum UA levels, lipid profile, renal and liver function were performed at each visit. eGFR was calculated with the Chinese abbreviated MDRD equation [6].

\section{Study Outcome}

The primary endpoint was change in renal function assessed as changes in eGFR. Secondary endpoints included change in pro- 
Table 1. Clinical characteristics of the patients at the time of renal biopsy

\begin{tabular}{lc}
\hline & Patients, $\mathrm{n}(\%)$ \\
\hline Male gender & $138(39.1)$ \\
Hypertension & $115(32.6)$ \\
Proteinuria $\leq 1 \mathrm{~g} / 24 \mathrm{~h}$ & $246(69.6)$ \\
$\quad>1 \mathrm{~g} / 24 \mathrm{~h}$ & $107(30.3)$ \\
$\quad>2 \mathrm{~g} / 24 \mathrm{~h}$ & $47(13.3)$ \\
$\quad>3 \mathrm{~g} / 24 \mathrm{~h}$ & $31(8.7)$ \\
Elevated Scr, >1.5 mg/dl & $85(24.1)$ \\
Macrohematuria & $90(25.5)$ \\
Hyperuricemia & $112(31.7)$ \\
Hypertriglyceridemia & $104(29.5)$ \\
Hypercholesterolemia & $106(30.0)$ \\
Obesity & $17(4.8)$ \\
Lee's grade (IV-V) & $163(46.2)$ \\
\hline
\end{tabular}

Table 2. Adjusted RR with 95\% CI for progression of IgAN (eGFR decline $\geq 50 \%$ or the initiation of renal replacement therapy or death) with respect to various clinical characteristics at the time of renal biopsy

\begin{tabular}{lccc}
\hline & RR & $95 \%$ CI & p value \\
\hline Hypertension & 1.2 & $1.1-1.4$ & 0.001 \\
eGFR $<60 \mathrm{ml} / \mathrm{min}$ & 3.7 & $1.4-7.1$ & $<0.001$ \\
Hyperuricemia & 2.5 & $1.5-6.1$ & 0.03 \\
Hypertriglyceridemia & 1.3 & $1.1-2.9$ & 0.04 \\
Lee's grade & 1.8 & $1.2-2.7$ & 0.02 \\
ACEI/ARB use & 0.4 & $0.1-0.8$ & 0.005 \\
\hline
\end{tabular}

teinuria, changes in BP (including antihypertension drug dosage), as well as adverse events.

\section{Statistical Analysis}

Power Calculation. This is the first pilot study to evaluate the effect of allopurinol on the clinical outcome in IgAN patients with hyperuricemia. To determine the sample size, we referenced the Siu et al. [10] study, which indicated that in general, $16 \%$ of the CKD patients in the allopurinol group reached the combined endpoints of significant deterioration in renal function and dialysis dependence compared with $46.1 \%$ patients in the control group. Under these assumptions and $10 \%$ of missing rate of follow-up, a sample size of 40 patients in each of the two treatment groups was needed to yield $80 \%$ power with an $\alpha$ level of 0.05 .

Data Analysis. Baseline characteristics of the retrospective cohort study and the randomized controlled trial are expressed as mean $\pm \mathrm{SD}$ for continuous variables and frequencies (percentages) for categorical variable. For the retrospective cohort study, the Cox proportional hazard regression model was used to assess the independent effect of hyperuricemia on the progression of IgAN. The results were expressed by using the adjusted relative risk (RR, or hazard ratio) and 95\% confidence interval (CI) for decline of eGFR $>50 \%$ the initiation of renal replacement therapy or death. The cumulative probabilities of renal survival were presented in Kaplan-Meier curves stratified by CKD stages and hyperuricemia, and survival analyses were based on the log-rank test.

For the prospective randomized controlled trial, comparison of various parameters at baseline between the two groups was performed by means of independent Student's t tests. Comparison of various parameters between baseline and the end of the study period was performed by means of paired Student's t tests. In addition, eGFR was log-transformed to obtain a better approximation of the normal distribution, and analyses of covariances (ANCOVAs) using per-protocol analysis were then performed with adjustment for the eGFR at baseline. Correlation between two variables was assessed by Pearson's rank correlation test. Fisher's exact test was carried out to analyze the difference between the modifications of antihypertensive drugs in the two groups. Twosided probability values are reported, with 0.05 taken as the level of statistical significance. All statistical analyses were performed using SPSS version 13.0 (SPSS Inc., www.spss.com).

\section{Results}

\section{Retrospective Cohort Study}

Baseline clinical characteristics of the patients are shown in table 1. Of 353 subjects, 282 (79.9\%) had an $\mathrm{eGFR} \geq 60 \mathrm{ml} / \mathrm{min} / 1.73 \mathrm{~m}^{2}$ and 71 (20.1\%) had an eGFR $<60 \mathrm{ml} / \mathrm{min} / 1.73 \mathrm{~m}^{2}$ at the time of the renal biopsy. The mean age of subjects at biopsy was $35.0 \pm 10.3$ years (range 19-67). Two patients had gout and 1 received allopurinol therapy.

The mean follow-up period was 5 years (range 12-98 months). 96 patients received an ACEI or ARB during the study period. 50 patients reached the primary endpoint during the study follow-up period, of which 12 received renal replacement therapy. Two died during the followup: 1 of uremia, another of cerebrovascular accident during hemodialysis. The cumulative 1-, 3-, and 5-year renal survival rates were 99,95 , and $86 \%$.

Cox proportional hazard regression analysis revealed the following parameters as independent risk factors for the progression of doubling of Scr level or the initiation of renal replacement therapy: hypertension $(\mathrm{RR}=1.2$, 95\% CI 1.1-1.4), eGFR $<60 \mathrm{ml} / \mathrm{min}$ at biopsy $(\mathrm{RR}=3.7$, 95\% CI 1.4-7.1), hyperuricemia ( $\mathrm{RR}=2.5$, 95\% CI $1.5-$ 6.1), hypertriglyceridemia ( $\mathrm{RR}=1.3,95 \% \mathrm{CI} 1.1-3.8)$, and higher Lee's histological grade ( $\mathrm{RR}=1.8,95 \% \mathrm{CI} 1.2-2.7)$. The usage of ACEI or ARB was independently related with a lower risk of renal progression $(\mathrm{RR}=0.4,95 \% \mathrm{CI}$ 0.1-0.8) (table 2). 


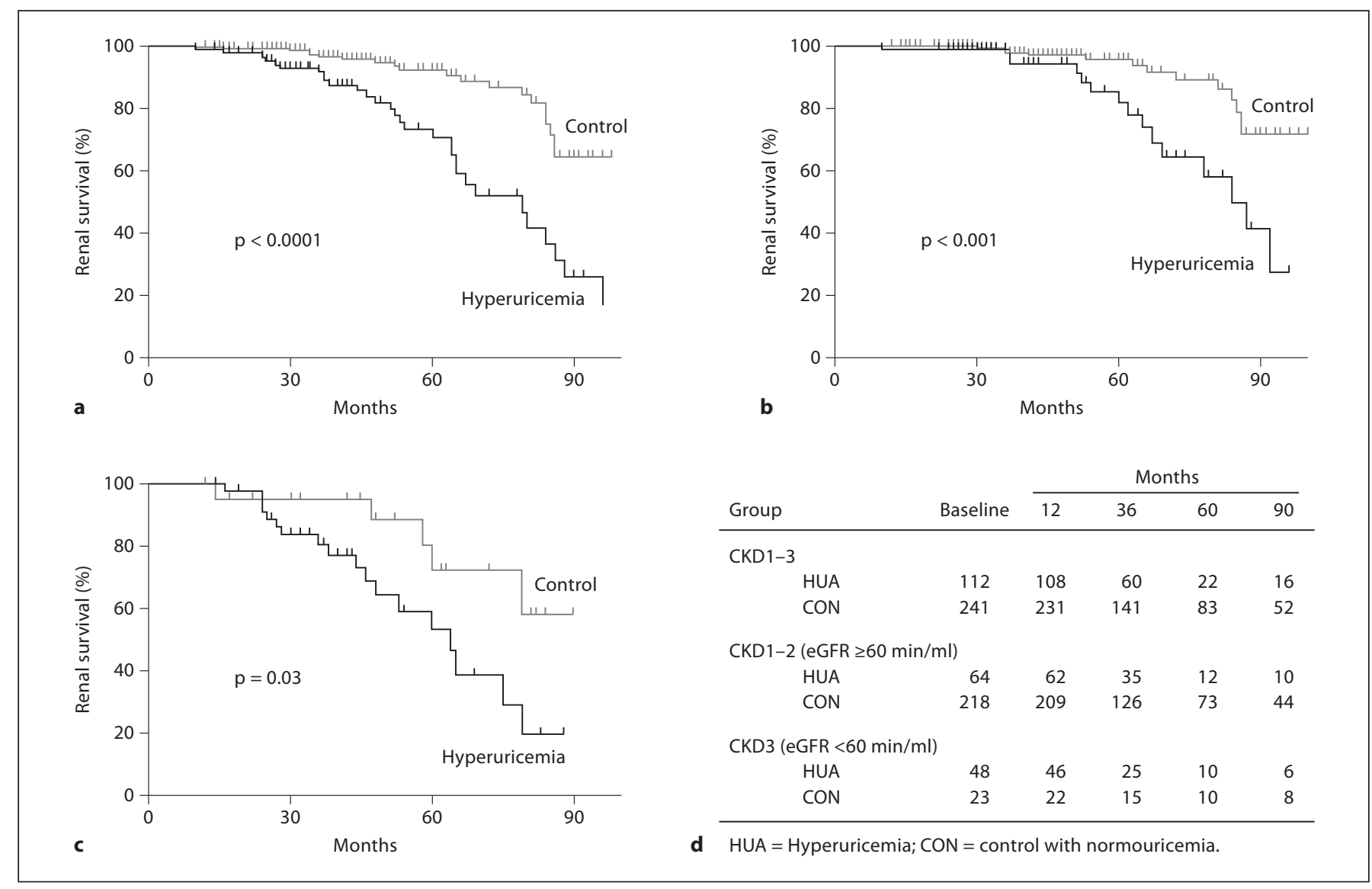

Fig. 1. a Cumulative probability of renal survival for all CKD (stages 1-3) patients in relation to UA at the time of renal biopsy. The differences in the renal survival of patients with or without hyperuricemia were statistically significant, $p<0.0001$. b Cumulative probability of renal survival for CKD (stage 1-2) patients with $\mathrm{eGFR} \geq 60 \mathrm{~min} / \mathrm{ml}$ in relation to UA at the time of renal biopsy. The differences in renal survival of patients with or without hyperuricemia were statistically significant, $\mathrm{p}<0.001$. c Cumulative probability of renal survival for CKD patients (stage 3 ) with eGFR $<60 \mathrm{~min} / \mathrm{ml}$ in relation to UA at the time of renal biopsy. The differences in the renal survival of patients with or without hyperuricemia were statistically significant, $p=0.03$. $\mathbf{d}$ Number of patients exposed to risk at the time point $(\mathbf{a}-\mathbf{c})$.
Of the 112 subjects with baseline hyperuricemia, 31 reached the primary endpoint compared to 19 of 241 control subjects without hyperuricemia (log-rank test: $\mathrm{p}<$ 0.0001 ; fig. 1a). The 1-, 3-, and 5-year renal survival rates were $98,92,68$ versus $100,97,92 \%$, respectively. After stratification by eGFR, the cumulative renal survival rates of patients with hyperuricemia versus those with normal UA remained significantly different regardless of initial eGFR level. Survival curves are shown for the CKD with eGFR $\geq 60 \mathrm{~min} / \mathrm{ml}$ (log-rank test: $\mathrm{p}<0.001$; fig. $1 \mathrm{~b}$ ) or CKD with eGFR $<60 \mathrm{~min} / \mathrm{ml}$ (log-rank test: $\mathrm{p}=0.03$; fig. 1c).

\section{Prospective Randomized Controlled Trial}

Forty patients were enrolled in the randomized clinical study with 21 in the allopurinol group 19 in the con- trol group (fig. 2). Baseline characteristics were comparable between the two groups (table 3). Three patients in the allopurinol group ( 2 due to ACEI/ARB usage and 1 due to doubling of Scr related to acute pneumonia) and 2 patients in the control group (1 due to usage of ACEI and 1 due to attack of gout) discontinued this study, and no patients were lost to follow-up.

\section{Primary Endpoint}

Renal Function

No difference was found in eGFR between the allopurinol treatment group and control group at the end of the study $\left(73.2 \pm 34.8\right.$ vs. $68.9 \pm 36.6 \mathrm{ml} / \mathrm{min} / 1.73 \mathrm{~m}^{2}, \mathrm{p}=$ 0.2 ). Also, no change of eGFR was found at the end of the study compared to the baseline in either the allopurinol 
Fig. 2. Study algorithm. Enrollment of patients, treatment assignments, and outcomes in the study.

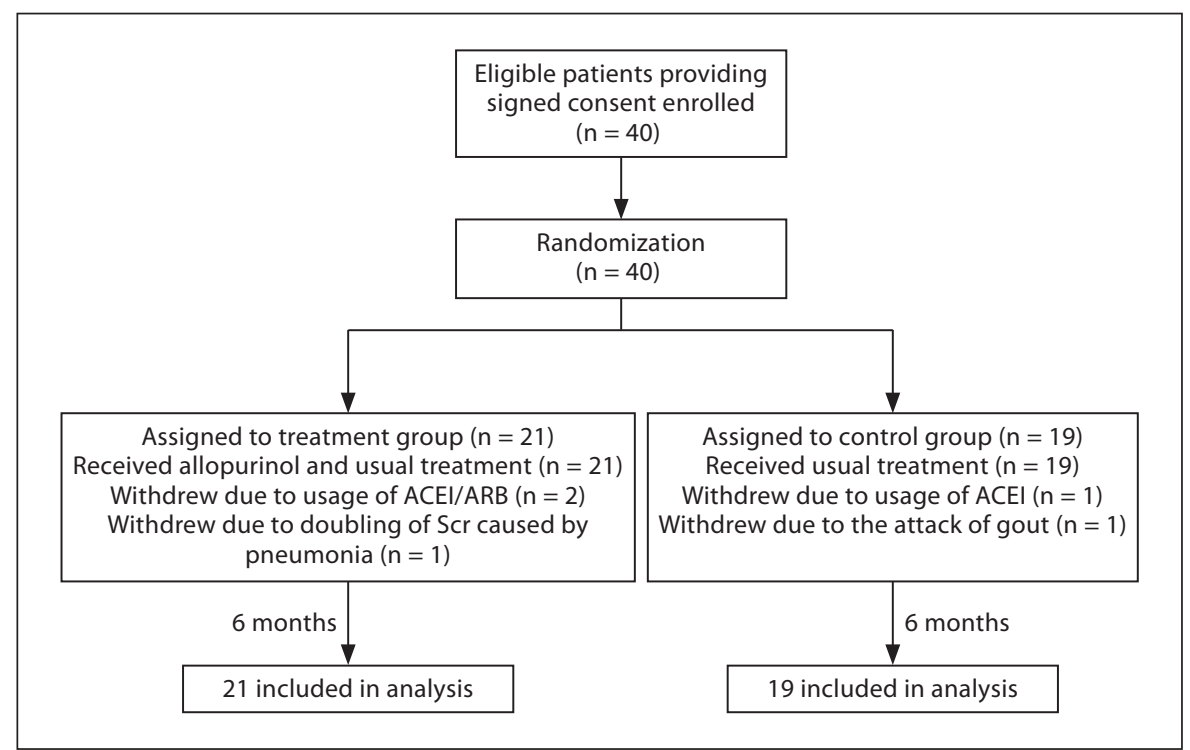

Table 3. Baseline characteristics in the treatment and control groups

\begin{tabular}{lccl}
\hline & $\begin{array}{l}\text { Allopurinol } \\
\text { group }\end{array}$ & $\begin{array}{l}\text { Control } \\
\text { group }\end{array}$ & $\begin{array}{l}\mathrm{p} \\
\text { value }\end{array}$ \\
\hline Patients, $\mathrm{n}$ & 21 & 19 & \\
Female:male & $8: 13$ & $10: 9$ & 0.4 \\
Age, years & $39.7 \pm 10.0$ & $40.1 \pm 10.8$ & 0.9 \\
Blood pressure & & & \\
$\quad$ Hypertension, $\mathrm{n}$ & 9 & 9 & \\
$\quad$ SBP, mm Hg & $139.1 \pm 23.8$ & $140.8 \pm 17.1$ & 0.8 \\
$\quad$ DBP, mm Hg & $88.1 \pm 14.3$ & $87.3 \pm 11.0$ & 0.8 \\
U pro/creat, mg/g & $959.9 \pm 1046.3$ & $835.9 \pm 599.7$ & 0.7 \\
Scr, mg/dl & $1.3 \pm 0.5$ & $1.4 \pm 0.5$ & 0.6 \\
eGFR, ml/min & $69.5 \pm 26.5$ & $63.6 \pm 27.5$ & 0.6 \\
UA, mg/dl & $7.9 \pm 1.1$ & $7.8 \pm 1.1$ & 0.7 \\
TG, mmol/l & $1.7 \pm 1.4$ & $1.6 \pm 0.9$ & 0.9 \\
TC, mmol/l & $4.8 \pm 0.9$ & $5.3 \pm 1.3$ & 0.2 \\
HDL, mmol/l & $1.2 \pm 0.3$ & $1.3 \pm 0.4$ & 0.1 \\
LDL, mmol/l & $3.1 \pm 1.0$ & $3.2 \pm 1.1$ & 0.9 \\
\hline
\end{tabular}

or control group (from $69.5 \pm 26.5$ to $73.2 \pm 34.8 \mathrm{ml} /$ $\mathrm{min} / 1.73 \mathrm{~m}^{2}$ for the allopurinol group and from $63.6 \pm$ 27.5 to $68.9 \pm 36.6 \mathrm{ml} / \mathrm{min} / 1.73 \mathrm{~m}^{2}$ for the control group, $p=0.2$ and $p=0.9$ respectively). eGFR tended to decrease in the allopurinol-treated patients in the first month compared to the control group, with the greatest decrease (9\%) among those subjects with baseline eGFR $<60 \mathrm{ml} /$ $\mathrm{min} / 1.73 \mathrm{~m}^{2}$ followed by recovery of renal function at lat- er time points (fig. 3a, b). In the control group, eGFR remained stable throughout the study. ANCOVA and adjusted means (95\% CI) for eGFR of the two groups before and after the therapy were $1.63(1.60,1.66)$ and $1.60(1.56$, $1.64), p=0.2$.

\section{Secondary Endpoints \\ Proteinuria}

There were no differences in the $\mathrm{U}_{\text {prot/creat }}$ ratio between the two groups, either at the baseline or follow-up (table 4). An increasing $\mathrm{U}_{\text {prot/creat }}$ ratio occurred in $48.5 \%$ of controls and in $31.8 \%$ of the allopurinol-treated group.

\section{Blood Pressure}

There were significant differences in requirements for BP treatment between the allopurinol and control group during the study $(\mathrm{p}=0.003)$. In the allopurinol treatment group, 7 of 9 patients had antihypertensive drugs reduced, including 1 patient who was able to stop antihypertensive medications. The remaining 2 patients underwent no change in antihypertensive therapy. In the control group, 3 patients increased their antihypertensive drugs and 6 patients continued their initial drug therapy; no patients decreased their antihypertensive regimen.

For patients with normal BP, treatment with allopurinol reduced serum UA compared to baseline ( $7.8 \pm 0.7$ vs. $5.3 \pm 0.8 \mathrm{mg} / \mathrm{dl}, \mathrm{p}<0.01, \mathrm{n}=9$ ) in association with a significant decrease in MAP $(92.9 \pm 10.1$ vs. $83.7 \pm 4.5$ $\mathrm{mm} \mathrm{Hg}$ pre- and post-allopurinol, respectively, $\mathrm{p}<0.01$ ). 


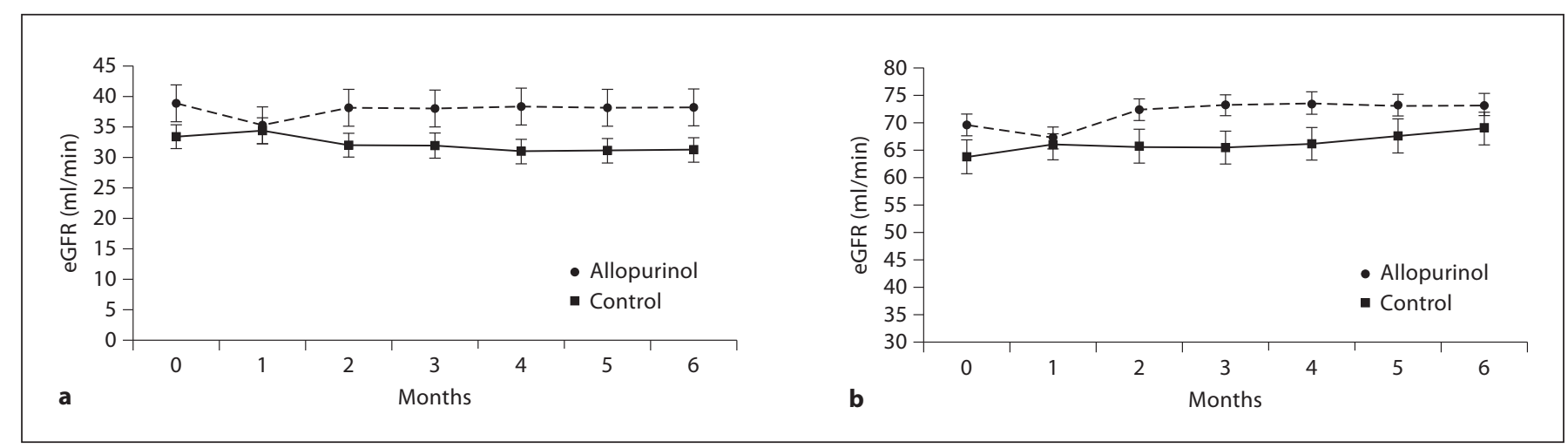

Fig. 3. a Baseline and repeated values of eGFR during the 6-month therapy for patients with eGFR $<60 \mathrm{ml} / \mathrm{min} / 1.73 \mathrm{~m}^{2}$ treated with allopurinol or control therapy. eGFR was not significantly changed at the end of 6 months. $\mathbf{b}$ Baseline and repeated values of eGFR during the 6-month therapy for patients treated with allopurinol or control therapy. eGFR was not significantly changed at the end of 6 months ( $p=0.2$ for allopurinol group vs. baseline value, $\mathrm{p}=0.9$ for control treatment group vs. baseline value and $\mathrm{p}=0.2$ compared with two treatment groups at the end of study, respectively). By the end of the first month, compared to baseline values before allopurinol treatment, eGFR was reduced (by $9.3 \pm$ $1.4 \%, \mathrm{p}=0.2$ ).

Table 4. Biochemical parameters of the treatment and control groups

\begin{tabular}{|c|c|c|c|c|}
\hline & \multicolumn{2}{|c|}{ Allopurinol group } & \multicolumn{2}{|l|}{ Control group } \\
\hline & baseline & last follow-up & baseline & last follow-up \\
\hline $\mathrm{U}_{\text {prot/creat }}, \mathrm{mg} / \mathrm{g}$ & $959.9 \pm 1,046.3$ & $1,219.3 \pm 1,063.6$ & $835.9 \pm 599.7$ & $1,170.4 \pm 951.9$ \\
\hline eGFR, mg/dl & $69.5 \pm 26.5$ & $73.2 \pm 34.8$ & $63.6 \pm 27.5$ & $68.9 \pm 36.6$ \\
\hline $\mathrm{UA}, \mathrm{mg} / \mathrm{dl}$ & $7.9 \pm 1.1$ & $5.7 \pm 0.7^{*}, \#$ & $7.8 \pm 1.1$ & $7.4 \pm 1.5^{* *}$ \\
\hline $\mathrm{TG}, \mathrm{mmol} / \mathrm{l}$ & $1.7 \pm 1.4$ & $1.5 \pm 0.8$ & $1.6 \pm 0.9$ & $1.7 \pm 0.8$ \\
\hline $\mathrm{TC}, \mathrm{mmol} / \mathrm{l}$ & $4.8 \pm 0.9$ & $4.6 \pm 0.8$ & $5.3 \pm 1.3$ & $5.2 \pm 1.2$ \\
\hline $\mathrm{HDL}, \mathrm{mmol} / \mathrm{l}$ & $1.2 \pm 0.3$ & $1.2 \pm 0.2$ & $1.3 \pm 0.4$ & $1.3 \pm 0.3$ \\
\hline LDL, mmol/l & $3.1 \pm 1.0$ & $2.9 \pm 0.8$ & $3.2 \pm 1.1$ & $3.3 \pm 1.4$ \\
\hline
\end{tabular}

Compared with the baseline: ${ }^{*} \mathrm{p}<0.001 ;{ }^{* *} \mathrm{p}=0.03$.

Compared with the control group at the end of the study: ${ }^{\#} \mathrm{p}<0.001$.

In control subjects with normal BP, serum UA slightly decreased compared to baseline $(7.9 \pm 1.6$ vs. $7.3 \pm 1.8$ $\mathrm{mg} / \mathrm{dl}, \mathrm{p}=0.06, \mathrm{n}=8$ ) without changes in MAP (93.7 \pm 5.4 vs. $93.8 \pm 4.1 \mathrm{~mm} \mathrm{Hg}, \mathrm{p}=0.9)$. A strong correlation was observed between serum UA and MAP $(r=0.388$, p $<0.001$; fig. 4).

\section{Biochemical Parameters}

Serum UA decreased in subjects treated with allopurinol from $7.9 \pm 1.1$ to $5.7 \pm 0.7 \mathrm{mg} / \mathrm{dl}(\mathrm{p}<0.001$; table 4).
Adverse Events

A slight elevation of liver function enzymes occurred in 4 patients in the treatment group and all recovered by reduction of the dosage of allopurinol.

\section{Discussion}

We performed a preliminary evaluation of the role of UA on the clinical outcome in Chinese IgAN. In the retrospective cohort study, hyperuricemia was shown to be a risk factor for renal progression independent of baseline 


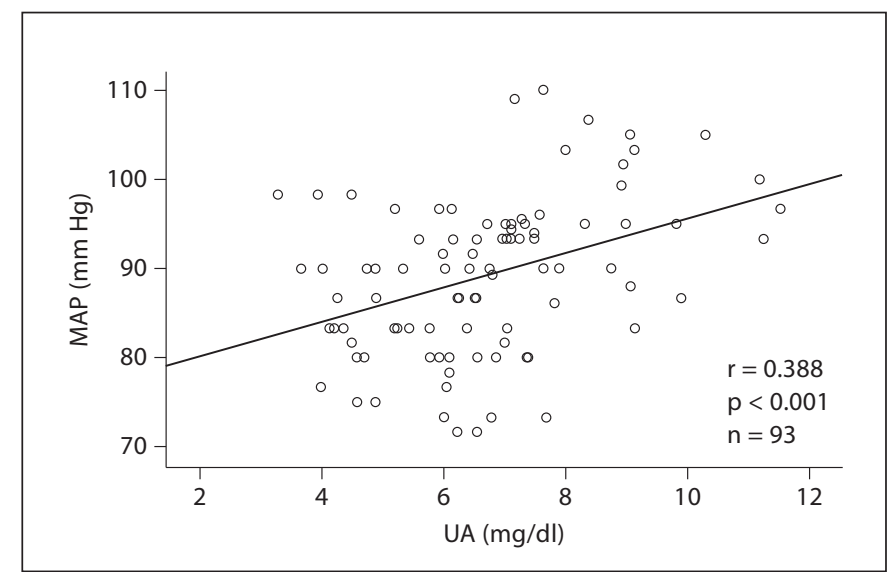

Fig. 4. Relationship between serum UA and MAP in patients without antihypertensive $(\mathrm{r}=0.388, \mathrm{p}<0.001)$.

eGFR. In addition, we conducted a prospective study with the main finding that lowering UA resulted in significant benefits on BP control.

UA appears to mediate its effects on the kidney via several mechanisms including activation of the renin-angiotensin system (RAS) as has been shown in in vitro studies [11], animal models [12] and as suggested by some clinical studies [13]. In addition, there is also increasing evidence that UA may have additional effects independent of the RAS. For example, UA potently inhibits endothelial nitric oxide in vitro via mechanisms including the stimulation of arginase (which degrades the substrate) [14], stimulating oxidants via NADPH oxidase (which scavenges nitric oxide) $[11,15]$, or by directly binding and inactivating nitric oxide [16]. UA has also been reported to stimulate thromboxane [17] and endothelin [18] production, and to have proinflammatory effects $[19,20]$.

Thus, we performed a pilot study to determine if lowering UA might have an effect on renal progression and $\mathrm{BP}$ in hyperuricemic subjects with IgAN. The main finding of the pilot trial was that the lowering of UA was associated with an improvement in BP control. There was a significant reduction in antihypertensive agents in allopurinoltreated subjects with baseline hypertension and there was also a significant fall in MAP in subjects not receiving antihypertensive therapy. A positive correlation between serum UA and MAP in patients not taking antihypertensive drugs was also demonstrated. In contrast, none of the control subjects with hypertension had a reduction in antihypertensive therapy and no change of MAP was observed in those subjects without hypertension. These data are con- sistent with increasing experimental [21] and clinical data [22] that suggest a role of UA in mediating hypertension.

Consistent with the reduction in systemic BP, allopurinol was associated with a transient worsening of renal function that was maximal in the first month and was greatest in subjects with baseline eGFR $<60 \mathrm{ml} / \mathrm{min} /$ $1.73 \mathrm{~m}^{2}$. This observation is similar to that observed with the initiation of ACEI in CKD and most likely represents reduced filtration from decreases in systemic and intraglomerular pressure. Indeed, Sanchez-Lozada et al. [23] reported that lowering UA could reduce glomerular pressure in animals with oxonic acid-induced hyperuricemic hypertension.

With regard to the primary outcome of the clinical trial and change in GFR, we found no benefit of allopurinol therapy on the eGFR at the end of the 6 months. This is most likely due to the short (6-month) duration of the study and the mild nature of the kidney disease of the recruited participants. Siu et al. [10] have reported that allopurinol can slow the progression of renal disease in subjects with CKD and asymptomatic hyperuricemia despite the fact that the majority were being treated with ACE inhibitors. A similar benefit was noted in a recent randomized study by Goicoechea et al. [24, 25], in which study subjects with CKD (stage 3 ) were randomized to allopurinol (100 mg/day) or control for 1 year, whereas renal function decreased in the controls and improved in the allopurinol group.

Our study is limited by the short duration and the small numbers of subjects. Nevertheless, an effect on BP was evident in this randomized, controlled trial. Another point: in vitro and vivo studies have shown that UA may mediate its effects on the kidney via several mechanisms including activation of the RAS. This is why we excluded the patients on ACEI/ARB in this pilot study. In a future RCT study, we would like to enroll all patients (with or without RAS blockade) and randomize them to receive treatment with ACEI/ARB alone, allopurinol alone or allopurinol combined with ACEI/ARB.

In conclusion, hyperuricemia is a risk factor for renal progression in $\operatorname{IgAN}$, and this effect is independent of baseline eGFR. We also report the first pilot study examining the effect of lowering UA with allopurinol in subjects with IgAN. While a benefit of allopurinol therapy was observed on BP, we were unable to observe any benefit of lowering UA on renal function or proteinuria at 6 months. However, longer duration of follow-up is necessary to better address the outcome of CKD progression. Further studies are warranted to determine if there is a role for UA-lowering therapy in the treatment of IgAN. 


\section{Acknowledgements}

This study was supported by grants from the Scientific and Technologic Committee of Guangdong Province (No. 2006A36001002, 2005B30701002), Guangdong Province Health Office (A2005189), and Guangdong Natural Science Foundation (6021368). This work has been made possible through Dr. Wei Chen's ISN Fellowship. Dr. Xueqing Yu was supported by Sun Yatsen University Clinical Research 5010 Program, the grant (No. 2010-76) from Guangdong province university academic and discipline development and the 973 project (2011CB50400050). Dr. Johnson was supported by NIH grants HL-68607 and DK-52121.

\section{Disclosure Statement}

Dr. Johnson has patent applications pending with the University of Florida and University of Washington related to lowering uric acid for subjects with hypertension or metabolic syndrome. The other authors declare no conflicts of interest.

\section{References}

1 Radford MG Jr, Donadio JV Jr, Bergstralh EJ, Grande JP: Predicting renal outcome in IgA nephropathy. J Am Soc Nephrol 1997;8:199207.

-2 Syrjanen J, Mustonen J, Pasternack A: Hypertriglyceridaemia and hyperuricaemia are risk factors for progression of IgA nephropathy. Nephrol Dial Transplant 2000;15:34-42.

-3 Ohno I, Hosoya T, Gomi H, Ichida K, Okabe $\mathrm{H}$, Hikita M: Serum uric acid and renal prognosis in patients with IgA nephropathy. Nephron 2001;87:333-339.

-4 Myllymaki J, Honkanen T, Syrjanen J, Helin H, Rantala I, Pasternack A, Mustonen J: Uric acid correlates with the severity of histopathological parameters in IgA nephropathy. Nephrol Dial Transplant 2005;20:89-95.

5 Lee SM, Rao VM, Franklin WA, Schiffer MS, Aronson AJ, Spargo BH, Katz AI: IgA nephropathy: morphologic predictors of progressive renal disease. Hum Pathol 1982;13: 314-322.

-6 Ma YC, Zuo L, Chen JH, Luo Q, Yu XQ, Li Y, $\mathrm{Xu}$ JS, Huang SM, Wang LN, Huang $W$, Wang M, Xu GB, Wang HY: Improved GFR estimation by combined creatinine and cystatin C measurements. Kidney Int 2007;72: 1535-1542.

7 Feig DI, Kang DH, Johnson RJ: Uric acid and cardiovascular risk. N Engl J Med 2008;359: 1811-1821.

8 Choo V: Who reassesses appropriate bodymass index for Asian populations. Lancet 2002;360:235.

-9 Chobanian AV, Bakris GL, Black HR, Cushman WC, Green LA, Izzo JL Jr, Jones DW, Materson BJ, Oparil S, Wright JT Jr, Roccella EJ: The Seventh Report of the Joint National Committee on Prevention, Detection, Evaluation, and Treatment of High Blood Pressure: the JNC 7 report. JAMA 2003;289: 2560-2572.

10 Siu YP, Leung KT, Tong MK, Kwan TH: Use of allopurinol in slowing the progression of renal disease through its ability to lower serum uric acid level. Am J Kidney Dis 2006; 47:51-59.
1 Corry DB, Eslami P, Yamamoto K, Nyby MD, Makino H, Tuck ML: Uric acid stimulates vascular smooth muscle cell proliferation and oxidative stress via the vascular renin-angiotensin system. J Hypertens 2008; 26:269-275.

12 Mazzali M, Kanellis J, Han L, Feng L, Xia YY, Chen Q, Kang DH, Gordon KL, Watanabe S, Nakagawa T, Lan HY, Johnson RJ: Hyperuricemia induces a primary renal arteriolopathy in rats by a blood pressure-independent mechanism. Am J Physiol 2002;282:F991F997.

13 Perlstein TS, Gumieniak O, Hopkins PN, Murphey LJ, Brown NJ, Williams GH, Hollenberg NK, Fisher ND: Uric acid and the state of the intrarenal renin-angiotensin system in humans. Kidney Int 2004;66:14651470 .

14 Zharikov S, Krotova K, Hu H, Baylis C, Johnson RJ, Block ER, Patel J: Uric acid decreases no production and increases arginase activity in cultured pulmonary artery endothelial cells. Am J Physiol Cell Physiol 2008; 295:C1183-C1190.

15 Sautin YY, Nakagawa T, Zharikov S, Johnson RJ: Adverse effects of the classical antioxidant uric acid in adipocytes: NADPH oxidase-mediated oxidative/nitrosative stress. Am J Physiol Cell Physiol 2007;293:C584C596.

16 Gersch C, Palii SP, Kim KM, Angerhofer A, Johnson RJ, Henderson GN: Inactivation of nitric oxide by uric acid. Nucleosides, Nucleotides Nucleic Acids 2008;27:967-978.

17 Kang DH, Nakagawa T, Feng L, Watanabe S, Han L, Mazzali M, Truong L, Harris R, Johnson RJ: A role for uric acid in the progression of renal disease. J Am Soc Nephrol 2002;13: 2888-2897.
18 Chao HH, Liu JC, Lin JW, Chen CH, Wu CH, Cheng TH: Uric acid stimulates endothelin-1 gene expression associated with NADPH oxidase in human aortic smooth muscle cells. Acta Pharmacol Sin 2008;29:1301-1312.

19 Kanellis J, Watanabe S, Li JH, Kang DH, Li P, Nakagawa T, Wamsley A, Sheikh-Hamad D, Lan HY, Feng L, Johnson RJ: Uric acid stimulates monocyte chemoattractant protein-1 production in vascular smooth muscle cells via mitogen-activated protein kinase and cyclooxygenase-2. Hypertension 2003;41: 1287-1293.

20 Kang DH, Park SK, Lee IK, Johnson RJ: Uric acid-induced C-reactive protein expression: implication on cell proliferation and nitric oxide production of human vascular cells. J Am Soc Nephrol 2005; 16:3553-3562.

21 Sanchez-Lozada LG, Tapia E, Santamaria J, Avila-Casado C, Soto V, Nepomuceno T, Rodriguez-Iturbe B, Johnson RJ, Herrera-Acosta J: Mild hyperuricemia induces vasoconstriction and maintains glomerular hypertension in normal and remnant kidney rats. Kidney Int 2005;67:237-247.

22 Brinker AD: Allopurinol and the role of uric acid in hypertension. JAMA 2009;301:270271.

23 Sanchez-Lozada LG, Tapia E, Avila-Casado C, Soto V, Franco M, Santamaria J, Nakagawa T, Rodriguez-Iturbe B, Johnson RJ, Herrera-Acosta J: Mild hyperuricemia induces glomerular hypertension in normal rats. Am J Physiol 2002;283:F1105-F1110.

24 Goicoechea M, de Vinuesa SG, Verdalles U, Ruiz-Caro C, Ampuero J, Rincon A, Arroyo D, Luno J: Effect of allopurinol in chronic kidney disease progression and cardiovascular risk. Clin J Am Soc Nephrol 2010;5:13881393.

25 Goicoechea M, Vinuesa SG, Verdalles U, Ruiz-Caro C, Ampuero J, Rincon A, Arroyo D, Luno J: Effect of allopurinol in chronic kidney disease progression and cardiovascular risk. Clin J Am Soc Nephrol 2010;5:13881393. 